\title{
Note on Possible Emergence Time of Newtonian Gravity
}

\author{
Lajos Diósi \\ Wigner Research Center for Physics \\ H-1525 Budapest 114, POB 49, Hungary
}

\begin{abstract}
If gravity were an emergent phenomenon, some relativistic as well as non-relativistic speculations claim it is, then a certain emergence time scale $\tau$ ? would characterize it. We argue that the available experimental evidences have poor time resolution regarding how immediate the creation of Newton field of accelerated mass sources is. Although the concrete theoretical model of gravity's 'laziness' is missing, the concept and the scale $\tau$ ? $\sim 1 \mathrm{~ms}$, rooted in an extrapolation of spontaneous wave function collapse theory, might be tested directly in reachable experiments.
\end{abstract}

tel/fax: +36-302956469/-13959151, e-mail: diosi.lajos@wigner.mta.hu

Physicists have always been speculating that gravity may emerge from a structure of deeper level. Gravity has been resisting to relativistic quantum field theories despite their robust success all over the past fifty-sixty years. If gravity itself is not a relativistic quantized field, it might be induced by them. According to Sakharov [1], the Casimir energy of quantized matter fields yields elastic forces on the background space-time, resulting in similar dynamics to Einstein's general relativity. This approach, its variants and refinements (cf., e.g., [2, 3] ) represent the main stream of 'emergent gravity' investigations. Alternative concepts [4 [6] relate Einstein's theory to thermodynamics and derive the gravitational force from the entropy. Other speculations postpone the relativistic aspects, abandon quantum field theory, but assume an intrinsic relationship between Newtonian (i.e., non-relativistic) gravity and quantum mechanics [713]. Some advocate phenomenological mechanisms for the emergence of the Newton interaction [11, 14, 15].

Our note restricts itself for emergence of the Newtonian, non-relativistic gravity. If this emergence is real at all, it must be characterized by a certain emergence time $\tau_{\text {? }}$ and the value of $\tau_{\text {? }}$ is expected to be longer than the typical time-scales of relativistic emergence. It could depend on the wave length but we further simplify our assumption as to look for a single time scale $\tau_{\text {? }}$. We mean that the gravitational field of an accelerated mass source shall not immediately follow the Newton law but with a delay of about $\tau_{\text {? }}$. Although the concrete model of emergence is missing, the option has been discussed recently [15] as a plausible consequence of the gravityrelated spontaneous wave function collapse theory $[8,10]$. The heuristic discussion concluded to the existence of a characteristic emergence time $\tau_{\text {? }} \sim 1 \mathrm{~ms}$.

As we said, our proposal is purposely non-relativistic but its relationship to the Einstein theory must be stated. A slight laziness, like $\tau_{\text {? }} \sim 1 \mathrm{~ms}$, in creating gravity would not invalidate the Einstein theory for the large scale dynamics of space-time. The only available experimental (indirect) evidence of gravitational waves confirms the radiation of the Hulse-Taylor binary pulsar at period $7.75 \mathrm{~h}$ [16], no way would they be influenced by our proposal.
Further relativistic predictions of the Einstein theory are light deflection, lensing and delay in the presence of gravity: these effects have been confirmed (cf. [17]) in the gravitational field of static or slowly moving sources of irrelevant acceleration to confront the above guess of $\tau_{\text {? }}$.

For the proposed short emergence time $\tau_{\text {? }}$, the peaceful coexistence with the above gravitodynamic evidences is comforting. Yet, we propose a concrete modification of Newton gravity which is even more reassuring: it reduces to the standard theory for purely gravitational manybody systems. Perhaps no Galilean invariant many-body model is able to capture a finite emergence time. Nevertheless, we know that standard field theories capture finite propagation times hence we tolerate the theoretical obstacles of finite emergence times. They might be relaxed in the framework of a future theory where certain 'fields' - dynamical and/or statistical — assist to massive bodies. For the time being, we propose a simplest phenomenology.

We start from the standard Newton law:

$$
\Phi(r, t)=\frac{-G M}{\left|r-x_{t}\right|},
$$

where $\Phi$ is the Newton potential at location $r$ and time $t$, created by the mass $M$ at location $x_{t}$ at the same time $t$. We propose the following retarded-smoothened version of the standard Newton potential:

$$
\Phi(r, t)=\int_{0}^{\infty} \frac{-G M}{\left|r-x_{t-\tau}\right|} \mathrm{e}^{-\tau / \tau_{?}} d \tau / \tau_{?},
$$

but this cannot be the full proposal because the value of $\Phi$ depends on the choice of the inertial frame. Suppose the source is free-falling in a certain, say, homogeneous external gravitational field. And suppose that we use the same free-falling reference frame. The equivalence principle, just the non-relativistic one, says that in the free-falling reference frame physics goes as if we were in a gravity-free inertial frame. If, furthermore, we use the co-moving frame where $\dot{x}_{t} \equiv 0$ then our proposal (2) reduces to the standard law (11). This result is plausible: if the source is at rest in an inertial (gravity-free) frame 
then its Newton potential is static, not delayed at all by the emergence time $\tau_{\text {? }}$. Therefore we require that our equation (2) be valid i) in the free-falling reference frame where $M \ddot{x}_{t}$ is equal to the non-gravitational forces and ii) in the $t$-dependent co-moving system where $\dot{x}_{t}=0$.

The second condition guarantees the Galilean boostinvariance of our proposal. The first condition (together with the second) guarantees that masses performing inertal motion solely under graviational forces would create the standard immediate Newton field, without the delay. Hence our modification of the Newton law does not influence the planetary dynamics. It influences systems with non-gravitational forces.

Can we then find evidences pro or contra our proposal in accomplished laboratory experiments on Newton theory? In a standard Cavendish experiment [18], a torsion balance measures the gravitational attraction produced by static source masses. Because of static sources, time-resolution is beyond the scope of the standard Cavendish experiments. Fortunately, there are Cavendish experiments with moving sources. In the Gundlach-Merkowitz experiment the sources are revolving and a time-resolution below $1 \mathrm{~min}$ seems available [19]. The re-analysis of the experimental data would put an upper limit on gravity's laziness $\tau_{\text {? }}$, stronger than ever. Furthermore, a precise measurement can be done at the gravity wave detectors [20], too. While they cannot resolve the gravity wave propagation time from a moving nearby source (e.g.: a spinning dumbbell), they would perfectly resolve the emergence time in (and much below) the range of $1 \mathrm{~s}$.

Let us apply our proposal to a laboratory source accelerated by non-gravitational forces. Eq. (2) has been postulated in the co-moving inertial frame where $\dot{x}_{t}=0$; in the laboratory system it acquires the boost $-\dot{x}_{t}$ :

$$
\Phi(r, t)=\int_{0}^{\infty} \frac{-G M}{\left|r-x_{t-\tau}-\dot{x}_{t} \tau\right|} \mathrm{e}^{-\tau / \tau_{?}} d \tau / \tau_{?} .
$$

The lowest order expansion in $\tau_{\text {? }}$ yields

$$
\Phi(r, t)=\frac{-G M}{\left|r-x_{t}\right|}\left(1+\frac{\ddot{x}_{t}^{r} \tau_{?}^{2} / 2}{\left|r-x_{t}\right|}\right) .
$$

The correction of the Newton law is proportional to the radial acceleration $\ddot{x}_{t}^{r}$ of the source. The emergent field $\Phi(r, t)$ is stronger/weaker if the source accelerates respectively toward/off the location $r$. If, e.g., the source is revolving at constant angular frequency $\Omega$ along a circle, the field in the center of the orbit is enhanced by the factor $1+\Omega^{2} \tau_{?}^{2} / 2$, valid for $\Omega \tau_{\text {? }} \ll 1$.

Let's summarize our work. We noticed that the timeresolution of available experimental data would not disclose a tiny temporal "laziness" $\tau_{\text {? }}$ of Newton gravity. We propose a delay time of the order of $\tau_{\text {? }} \sim 1 \mathrm{~ms}$, coming from speculations on spontaneous wave function collapse. A minimalist modification of the Newton law captures the delay $\tau_{\text {? }}$ in such a way that the dynamics of purely gravitational motion remains the standard one. Our proposal modifies the Newton field of sources accelerated by non-gravitational forces that is typical in laboratory experiments. Even if the theoretical background of a possible emergence time $\tau_{\text {? }}$ is vague at the moment, reachable laboratory experiments should answer if $\tau_{\text {? }}$ can be that big as $1 \mathrm{~ms}$. They would easily push the upper limit on $\tau_{\text {? }}$ much below $1 \mathrm{~ms}$. Or, they might in principle find new physics with $\tau_{\text {? }} \sim 1 \mathrm{~ms}$ (or with even bigger one), confirming or at least encouraging the related quantum theoretical speculations [15].

Support by the Hungarian Scientific Research Fund under Grant No. 75129 and support by the EU COST Action MP100 are acknowledged. The author thanks Tamás Geszti and Péter Király for interesting discussions.

[1] A.D. Sakharov, Dokl. Akad. Nauk. SSSR 177, 70 (1967); Gen. Rel. Grav. 32365 (2000).

[2] S.L. Adler, Rev. Mod. Phys. 54, 729 (1982).

[3] M. Visser, Mod. Phys. Lett. A 17977 (2002).

[4] T. Jacobson, Phys. Rev. Lett. 75, 1260 (1995).

[5] E. P. Verlinde, JHEP 04, 029 (2011).

[6] T. Padmanabhan, Rep. Prog. Phys. 736901 (2010).

[7] L. Diósi and B. Lukács, Annln. Phys. 44488 (1987); L.Diósi: Quantum measurement and gravity for each other, p299 in: Quantum Chaos - Quantum Measurement, eds.: P.Cvitanovic, I.Percival and A. Wirzba (Kluwer, Dordrecht, 1992).

[8] L. Diósi, Phys. Lett. 120A 377 (1987); L. Diósi, Phys. Rev. A40, 1165 (1989).

[9] K.R.W. Jones, Aust. J. Phys. 48, 1055 (1995).

[10] R. Penrose, Gen. Rel. Grav. 28, 581 (1996);

[11] S. De Filippo and F. Maimone, Phys. Rev. D 66044018 (2002); F. Maimone, G. Scelza, A. Naddeo and V. Pelino, Phys. Rev A 83062124 (2011).

[12] T. Geszti, Phys. Rev. A69, 032110 (2004).

[13] J. Van Wezel, T. Oosterkamp, J. Zaanen, Phil. Mag. 88 1005 (2008); J. Van Wezel and J. Van der Brink, Phil. Mag. 881659 (2008).

[14] L. Diósi and T.N. Papp, Phys. Lett. 373A 3244 (2009); L. Diósi, J. Phys. Conf. Ser. 174012002 (2009).

[15] L. Diósi: Gravity-related wave function collapse: Is superfluid He exceptional?, arXiv:1302.5364 L. Diósi: Gravity-related wave function collapse: mass density resolution, arXiv:1302.5365.

[16] R. V. Wagoner, Astrophys. J. Lett. 196, L63 (1975).

[17] I. Ciufuloni and J.A. Wheeler: Gravitation and Inertia (Princeton University Press, Princeton, 1995).

[18] G.G. Luther and G.R. Towler, Phys. Rev. Lett. 48, 121 (1982).

[19] J.H. Gundlach and S.M. Merkowitz, Phys. Rev. Lett. 85, 2869 (2000).

[20] B.P. Abbott, et al. (LIGO Scientific Collaboration), Rep. Prog. Phys. 72, 076901 (2009); T. Accadia et al. (VIRGO Scientific Collaboration), J. Phys.: Conf. Ser. 203, 012074 (2010). 